\title{
Analysis of Acoustic Emission Data for Bearings subject to Unbalance
}

\author{
Seyed A. Niknam ${ }^{1}$, Tomcy Thomas ${ }^{1}$, J. Wesley Hines ${ }^{2}$, and Rapinder Sawhney ${ }^{1}$ \\ ${ }^{1}$ Department of Industrial and Systems Engineering, University of Tennessee, Knoxville, TN 37996, USA \\ sniknam@utk.edu \\ tomcy@utk.edu \\ sawhney@utk.edu \\ ${ }^{2}$ Department of Nuclear Engineering, University of Tennessee, Knoxville, TN 37996, USA \\ jhines2@utk.edu
}

\begin{abstract}
Acoustic Emission (AE) is an effective nondestructive method for investigating the behavior of materials under stress. In recent decades, AE applications in structural health monitoring have been extended to other areas such as rotating machineries and cutting tools. This research investigates the application of acoustic emission data for unbalance analysis and detection in rotary systems. The AE parameter of interest in this study is a discrete variable that covers the significance of count, duration and amplitude of AE signals. A statistical model based on Zero-Inflated Poisson (ZIP) regression is proposed to handle overdispersion and excess zeros of the counting data. The ZIP model indicates that faulty bearings can generate more transient wave in the $\mathrm{AE}$ waveform. Control charts can easily detect the faulty bearing using the parameters of the ZIP model. Categorical data analysis based on generalized linear models (GLM) is also presented. The results demonstrate the significance of the couple unbalance.
\end{abstract}

\section{INTRODUCTION}

Acoustic emission (AE) is defined as transient elastic waves generated due to localized physical changes in a solid material under mechanical or thermal stresses (Tan et al., 2007). AE is also referred to as the practical non-destructive technology to investigate the behavior of stressed materials using the transient elastic waves. The major advantage of this technology is its sensitivity to capture surface and subsurface micro-damage. It has been proven that in some cases AE can ensure superiority over vibration-based monitoring systems in early fault detection (Tan et al., 2007, Alghamd \& Mba, 2006, Tandon \& Mata, 1999,

Niknam et al. This is an open-access article distributed under the terms of the Creative Commons Attribution 3.0 United States License, which permits unrestricted use, distribution, and reproduction in any medium, provided the original author and source are credited.
Eftekharnejad \& Mba, 2009). In case of rolling-element bearings, AE detects the fault earlier compared to other technologies (Mba \& Rao, 2006, Yoshioka \& Fujiwara 1982). Significant changes in vibration signatures can be observed when the remaining operational life of a bearing is very short. Hence, AE offers good potential for prognostic capabilities. Additionally, insensitivity to structural resonance and mechanical background noise gives $\mathrm{AE}$ an additional advantage over typical vibration-based monitoring systems. However, AE signals may suffer severe attenuation and reflections due to sensor positioning and machine complexity. $\mathrm{AE}$ is a well-established diagnostic method for static structures. In recent decades, $\mathrm{AE}$ applications in structural health monitoring have been extended to other areas such as rotating machineries and cutting tools (Niknam \& Liao, 2011). The readers are referred to ISO 22096:2007 for the general principles of AE application.

$\mathrm{AE}$ hit is defined as the process of detecting and measuring an AE signal on a channel (PAC, 2007). The fundamental features of the AE hit include amplitude, duration, count, and rise time. These parameters can be used to provide additional signal features such as root mean square (RMS), AE cumulative event count, counts to peak, rise time slope, crest factor and Kurtosis (Alghamd \& Mba, 2006, Tandon \& Mata, 1999, K. Miyachika et al. 1995, He et al. 2010, Bansal et al. 1990, Choudhury \& Tandon, 2000). Figure 1 illustrates the diagram of $\mathrm{AE}$ hit feature extraction (PAC, 2007).

AE count is defined as the number of times the signal crosses the threshold in an $\mathrm{AE}$ hit. In the literature, this parameter is also known as ring down count or threshold crossing count. In effect, AE counts imply the existence of a transient wave in the AE waveform. One major drawback of this parameter is its dependence on the threshold level. It is to be noted that the average counts is a measure of $\mathrm{AE}$ intensity i.e. the size of the emission signals detected. 
Furthermore, AE counts divided by duration gives the average frequency of the signal. The next section provides more details of the application of $\mathrm{AE}$ count for fault detection. The parameter of interest in this study is PACenergy (PAC is the registered trademark of Physical Acoustics Corporation). PAC-energy is a 2-byte parameter derived from the integral of the rectified voltage signal over the duration of AE hit (PAC 2007). The unit of PAC-energy is micro-volt-seconds per count and the range is 0 to 65535 in each $\mathrm{AE}$ hit. Therefore, this parameter is a discrete random variable that covers the significance of count, duration and peak amplitude. Thus, the statistical models for count data can be applied for the PAC-energy.

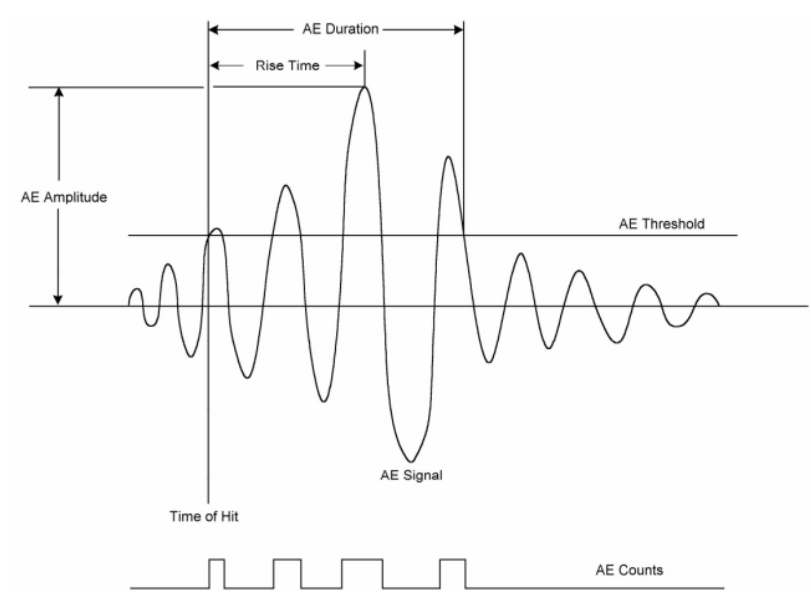

Figure 1. Diagram of AE hit feature extraction (PAC 2007)

This research attempts to provide effective diagnostic and prognostic algorithms for wind turbine drive trains. In practice, rotor unbalance is a major concern in wind turbine reliabilities. Rotor unbalance in wind turbines is caused by manufacturing defects, accumulated damage to the rotor blades, and non-uniform accumulation of ice, dust, and moisture (Lu et al. 2009, Hyers et al., 2006). Rotor unbalance has an important influence on the pitch mechanism, the yaw brakes and the variations in the rotor speed which can even affect the output power waveform. Furthermore, rotor unbalance imposes additional vibrations of the nacelle, tower and the drivetrain components ( $\mathrm{Lu}$ et al. 2009, Hyers et al., Hameed et al., 2009). It may also shorten the life of the rotor blades. In particular, this research investigates the application of AE data in analyzing unbalanced rotary systems. Categorical data analysis based on generalized linear models (GLM) is presented in this study. The objective of this analysis is to provide a meaningful interpretation of the test variables' effect on $\mathrm{AE}$ features. In addition, a statistical model based on ZeroInflated Poisson (ZIP) regression is proposed to handle over-dispersion and excess zeros of the counting data. The ZIP model considers the data set as a mixture of processes that generates only zeros or non-negative integers.
The remainder of the paper is organized as follows: Section 2 provides the background for unbalanced systems, a review of using AE count in fault detection, and modeling of count data. Section 3 presents a detailed description of the experimental setup and the test procedure. Section 4 provides the analytical results and discussions. Finally, section 5 concludes this paper.

\section{BACKGROUND}

\subsection{AE count}

Traditionally, AE count has been widely used in the literature as condition indicator. Tandon and Mata (1999) realized that defects in gear systems would result in broader distribution of $\mathrm{AE}$ counts and peak amplitude. They claimed that $\mathrm{AE}$ counts showed better results than other $\mathrm{AE}$ parameters in gear defect detection. Miyachika et al. (1995) postulates that $\mathrm{AE}$ cumulative event count increases with crack growth in the case-hardened gears. Tandon and Nakra (1990) considered AE counts for condition monitoring of radially loaded ball bearing. They observed a direct relationship between $\mathrm{AE}$ count and speed in case of outer race defect. The results showed that $\mathrm{AE}$ count is a practical indicator for defects less than $250 \mu \mathrm{m}$ in diameter. However, Morhain and Mba (2003) declared the AE count is able to detect large defects up to $15 \mathrm{~mm}$ in lengths and $1 \mathrm{~mm}$ in width. This study also emphasized the sensitivity of AE counts to the level and grade of lubricant. AE parameter analysis for tool condition monitoring has received attentions mainly for real time applications. AE count rate was introduced as a reliable parameter for monitoring tool wear during turning, although AE signals highly depend on process parameters (Li 2002, Sharma et al. 2007). Carpinteri et al. (2007) applied AE technology to monitor concrete and masonry buildings. The cumulative number of counts was found to be informative for analyzing the evolution of cracks and determining the released strain energy. Carpinteri et al. (2007) observed that the maximum counting of $\mathrm{AE}$ corresponds to the maximum velocity of crack propagation. Considering the proven usefulness of AE count for various $\mathrm{AE}$ applications, it seems an appropriate modeling and analysis of $\mathrm{AE}$ count or its derivatives would lead to fruitful diagnostic. As mentioned earlier, PACenergy is a function of AE counts and it will be used for the statistical modeling in this study.

\subsection{Modeling count data}

In the literature, linear regression models have been primarily utilized to correlate the $\mathrm{AE}$ signatures and the physical features of interest. Traditional regression (nonBayesian) methods have been widely used to model count data in both natural and social sciences. In effect, the response variable in such models is a nonnegative integer. The most common regression-based count data model is Poisson GLM which is an extension of ordinary least 
squares regression and agrees with distributions from the exponential family. The Poisson GLM has the form

The log link is specified by

$$
y \sim \operatorname{Poisson}(\lambda)
$$

$$
\log \lambda=\beta_{0}+\sum_{i} \beta_{i} x_{i}
$$

The logistic model where $p$ is the probability of success is given by

$$
y \sim \operatorname{Binomial}(p, n)
$$

The logit link is given by

$$
\operatorname{logit} p=\log \frac{p}{1-p}=\beta_{0}+\sum_{i} \beta_{i} x_{i}
$$

The major drawback of Poisson regression is the restrictive assumption of equality between the variance and the mean i.e. equidispersion (Liu \& Cela 2008, Lambert 1992, Guikema \& Coffelt 2008). On the other hand, overdispersion occurs when the variance is greater than the mean. The overdispersion can be handled through negative binomial GLM. Generalized linear mixed models (GLMM) add an error term to GLM which is not part of our discussion.

In practice, depending on the preset reference threshold value, there may be a high frequency of zero counts in the $\mathrm{AE}$ signals, which indicates excess zeroes. In this case, a simple Poisson regression would not satisfactorily fit the data. Zero-inflated Poisson (ZIP) model, introduced by Lambert (1992), fittingly handles overdispersion and excess zeros. Principally, ZIP model considers the data set as a mixture of a process that generates only zeros and a process that generates counts from a Poisson or a negative binomial model. In other words, ZIP models calculate the probability (p) of having observations of 0 . Therefore, 1-p would be the probability of having non-negative integers. Therefore the count response can be written as

$$
\begin{array}{ll}
Y_{i} \sim 0 & \text { with probability } p_{i} \\
Y_{i} \sim \operatorname{PoisSon}\left(\lambda_{i}\right) & \text { with probability } 1-p_{i}
\end{array}
$$

Thus,

$$
\begin{gathered}
Y_{i}=0 \text { with probability } p_{i}+\left(1-p_{i}\right) e^{-\lambda_{i}} \\
Y_{i}=k \text { with probability }\left(1-p_{i}\right) e^{-\lambda_{i}}\left(\lambda_{i}\right)^{k} / k !
\end{gathered}
$$

Consider the following regression models for

$$
\begin{gathered}
\lambda=\left[\lambda_{1}, \lambda_{2}, \ldots, \lambda_{n}\right]^{\prime} \text { and } \mathrm{p}=\left[p_{1}, p_{2}, \ldots, p_{n}\right]^{\prime}: \\
\ln (\lambda)=\mathbb{B} \beta, \quad \operatorname{logit}(\mathrm{p})=\ln \left(\frac{\mathrm{p}}{1-\mathrm{p}}\right)=\mathbb{G} \gamma
\end{gathered}
$$

where $\mathbb{B}$ and $\mathbb{G}$ are covariate matrices (design matrices), $\beta$ and $\gamma$ are regression coefficients.

Accordingly, the log-likelihood function is

$$
\begin{gathered}
L(\beta, \gamma \mid \mathrm{y})=\sum_{y_{i}=0}^{n} \ln \left(e^{\mathbb{G}_{i} \gamma}+\exp \left(-\left(e^{\mathbb{B}_{i} \beta}\right)\right)\right. \\
-\sum_{i=1}^{n} \ln \left(1+e^{\mathbb{G}_{i} \gamma}\right)+\sum_{y_{i}>0}^{n}\left(y_{i} \mathbb{B}_{i} \beta-e^{\mathbb{B}_{i} \beta}\right)-\ln \left(y_{i} !\right)
\end{gathered}
$$

By using $\ln (\lambda)=\mathbb{B} \beta$ and $\operatorname{logit}(\mathrm{p})=\ln \left(\frac{\mathrm{p}}{1-\mathrm{p}}\right)=\mathbb{G} \gamma$ we conclude

$$
\lambda_{i}=\exp \left(\mathbb{B}_{i} \beta\right), \text { and } p_{i}=\frac{\exp \left(\mathbb{G}_{i} \gamma\right)}{1+\exp \left(\mathbb{G}_{i} \gamma\right)}
$$

Hence, using the delta method, the variances of $\hat{p}$ and $\hat{\lambda}$ are

$$
\begin{gathered}
\operatorname{cov}\left(\hat{\lambda}_{i}, \hat{p}_{i}\right)=\left[\left[\frac{\partial \lambda_{i}}{\partial \beta}\right]^{\prime}\left[\frac{\partial p_{i}}{\partial \gamma}\right]^{\prime}\right] \operatorname{cov}(\hat{\gamma}, \hat{\beta})\left[\left[\frac{\partial \lambda_{i}}{\partial \beta}\right]^{\prime}\left[\frac{\partial p_{i}}{\partial \gamma}\right]^{\prime}\right]^{\prime} \\
\text { and } \hat{\sigma}_{0\left(\hat{p}_{i}\right)}=\sqrt{\operatorname{var}\left(\hat{p}_{i}\right)}, \hat{\sigma}_{0\left(\hat{\lambda}_{i}\right)}=\sqrt{\operatorname{var}\left(\hat{\lambda}_{i}\right)}
\end{gathered}
$$

\subsection{Unbalanced rotary systems}

The lack of balance causes excessive vibration in rotary systems. Vibrations impose centrifugal force and oscillatory force. For wind turbines, unbalance occurs primarily due to uneven material deposit on the rotor about its rotating centerline. High vibration amplitude at the rotating speed is the primary indicator of this fault. Other sources of unbalance include imperfect manufacturing and operational changes. Bearings are a significant contributor to unbalance in rotary system because of internal clearance and run-out. In effect, the existence of heavy spot in rotors leads to shaft bending and cyclical forces on bearing. Both centrifugal and oscillatory forces affect the bearing life. The magnitude of centrifugal force can be obtained by

$$
F_{c}=m r \omega^{2}
$$

where $\mathrm{m}$ represents mass, $\omega$ represents the angular speed and $r$ is the radius from center of rotation.

There are various types of unbalance based upon the relation between the center of gravity (CG) and the heavy spot. In static unbalance, the CG and the heavy spot are in the same plane. Couple unbalance occurs due to existence of two equal heavy spots which are 180 degrees apart. In this case, the shaft axis intersects the principal mass at the CG. Couple unbalance imposes radial force on bearings. Dynamic unbalance is the most common type of unbalance and it represents a combination of static and couple unbalance. In essence, balancing treats the cause rather than symptom. Balancing may involve the followings: correction of mass distribution, creating centrifugal force, changing orientation of parts and adding/removing mass from non-rotating part (Wowk 1995). These actions would provide mass symmetry, change the center of gravity, and affect bending moments. It is important to note that cyclical forces on bearings measured through vibration analysis are utilized for balancing. However, in this research, the magnitudes of the 
balancing masses were found graphically. Details pertaining to the unbalance disks are presented in the next section.

\section{EXPERIMENTAL SET-UP AND PROCEDURE}

A multi-purpose test rig was designed and developed to simulate the drive train of wind turbines. Figure 2 shows the test rig. A WindMax $2 \mathrm{~kW}$ wind turbine generator was used in this setup. The test rig's motor provided a rotational speed in the range of 10- $1760 \mathrm{round} / \mathrm{min}$. The control drive along with a LabVIEW-based program allowed the motor to provide variable speed according to a pre-defined speed pattern. Other main components of the test rig include a single phase planetary gearbox, support bearings, and two test bearings of different size.

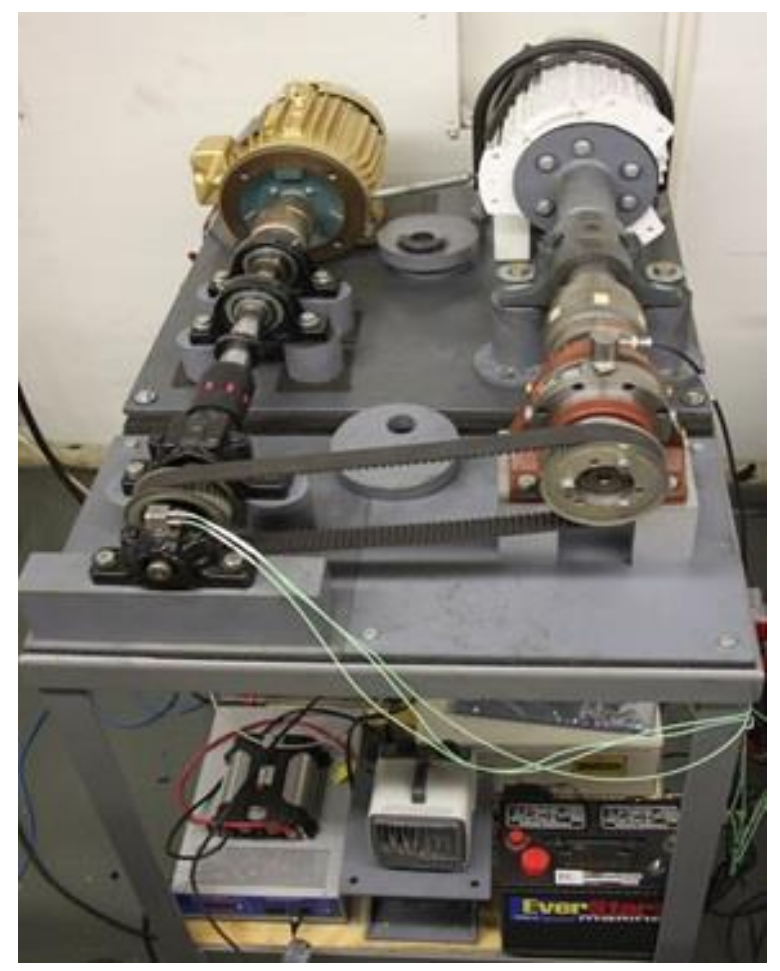

Figure 2. Test Rig

The test bearing used was a SKF self-aligning ball bearing type 1205 ETN9 (Figure 4). The characteristics of the test bearing are as follows: internal (bore) diameter $25 \mathrm{~mm}$, external diameter $52 \mathrm{~mm}, 26$ rollers, and diameter of roller $7.4 \mathrm{~mm}$. To make a faulty bearing, a groove with the width of $0.5 \mathrm{~mm}$ and the maximum height of $1.41 \mathrm{~mm}$ was made by using electrical discharge machine on the inner race of a bearing (Figure 4). Prior to the main tests, two bearings were run for 20 hours at the speed of $1700 \mathrm{rpm}$ i.e. over 2 million cycles. These bearings will be called "used bearings" in the analysis. It is important to note that all the bearings were used in dry condition. One reason for this is to ensure that the used bearings will not remain as good as new bearing after 2 million cycles.
To impose the unbalance force two uniform disks with the diameter of 6 inch and the thickness of 1 inch were used. To be able to attach the disks to the shaft and also change the angle of the disks, a shaft collar with a thickness of 0.5 inch was welded to each disk (Figure 3). An off-center hole with the diameter of 1.375 inch was then made for each disk as shown in Figure 5.

The mass of the disk with and without the hole were approximately 8 pounds and 7.6 pounds respectively. The new center of gravity moved off-center about 0.078 inch. The disks could be placed between the test bearings as shown in Figure 2. Consequently, several different sets of experiments could be performed by changing the number and orientation of the disks. In this study, the following loading configurations were used: no disk, one disk, two disks with an angle of zero, two disks with an angle of 120 and two disks with an angle of 180 namely couple unbalance.

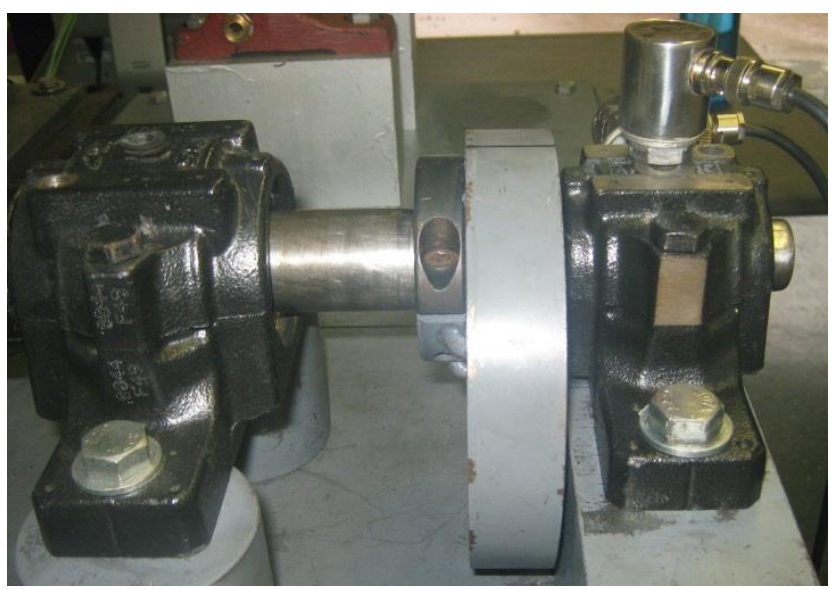

Figure 3. Sensor placement and an unbalance disk
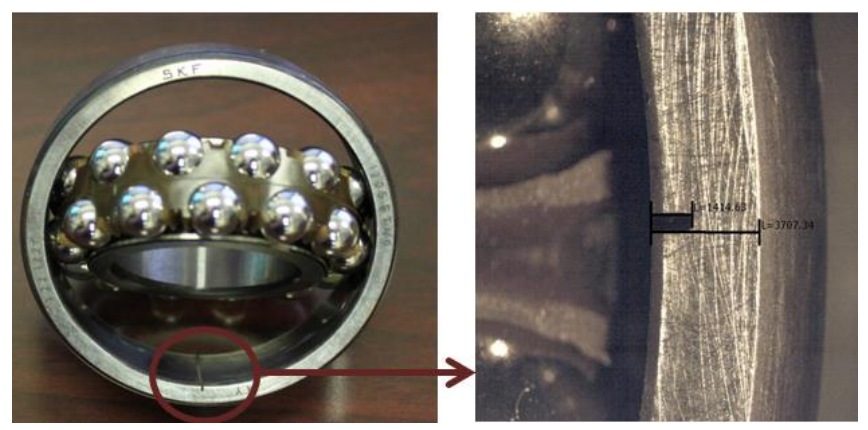

Figure 4. SKF 1205 ETN9 with a seeded fault

In this study, three major variables were considered: (1) bearing type, (2) shaft speed and (3) unbalance. Table 1 shows different categories of the variables. As shown in the table, three speed levels, three bearing types, and 5 loading patterns were designed for the experiments. 


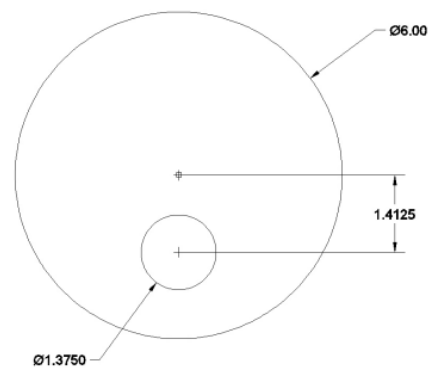

Figure 5. Disk with off-center hole

\begin{tabular}{|c|c|c|}
\cline { 2 - 3 } \multicolumn{1}{c|}{} & Settings & Category \\
\hline \multirow{4}{*}{ Unbalance } & No Disk & 1 \\
\cline { 2 - 3 } & 1 Disk & 2 \\
\cline { 2 - 3 } & 2 Disk w $00^{\circ}$ & 3 \\
\cline { 2 - 3 } & 2 Disk w $120^{\circ}$ & 4 \\
\cline { 2 - 3 } & 4 Disk w $180^{\circ}$ & 5 \\
\hline \multirow{3}{*}{ Bearing } & New & 1 \\
\cline { 2 - 3 } & Used & 2 \\
\cline { 2 - 3 } & Faulty & 3 \\
\hline \multirow{3}{*}{$\begin{array}{c}\text { Shaft Speed } \\
\text { (rpm) }\end{array}$} & 150 & 2 \\
\cline { 2 - 3 } & 300 & 3 \\
\cline { 2 - 3 } & 450 & \\
\hline
\end{tabular}

Table 1. Test Data Description

A 2-channel PCI-2 based AE system was utilized for data acquisition. The PAC R15I-AST acoustic sensor was used. This sensor has an operating frequency range of 80-200 $\mathrm{kHz}$. The sensor housing contains a filter and an integral preamplifier of $40 \mathrm{~dB}$. With an improved 18 bit analogue to digital conversion scheme, PCI-2 board provides a sampling rate of up to $40 \mathrm{MHz}$ and a dynamic range of more than 85 $\mathrm{dB}$. This data acquisition system is able to record up to 65535 counts per hit. The data acquisition was performed with a sampling rate of 5 mega points per seconds. The upper and lower limit of filter was set to $10 \mathrm{KHz}$ and $1 \mathrm{MHz}$ respectively. The sensors were placed on the bearing housing as shown in Figure 3. The sensor which was placed on the top provided more data than the sensor which was on the side of the housing. In essence, one can expect less data from the side sensor due to the interfaces that exist between the source and the side sensor. In figures 6 and 7 the left graph shows the energy collected from the top sensor and the graph on the right shows the energy collected from the side sensor. As shown in these figures, data from the side sensor is not really helpful. In Figure 6 the seeded fault was exactly below the top sensor. Experimenting various position of the seeded fault, it was realized that placing the fault exactly below the top sensor provides more energy.

In Figure 7 the seeded fault was placed 90 degrees apart from the top sensor and 180 degrees apart from the side sensor. For our analysis, only the data collected from the top sensor was utilized. Moreover, the movement of the bearing with inside the housing was negligible.
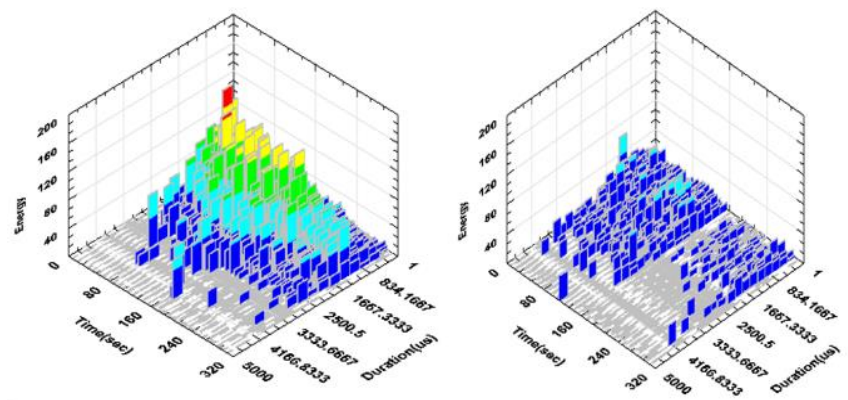

Figure 6. PAC-energy from the sensors while the fault was under the top sensor
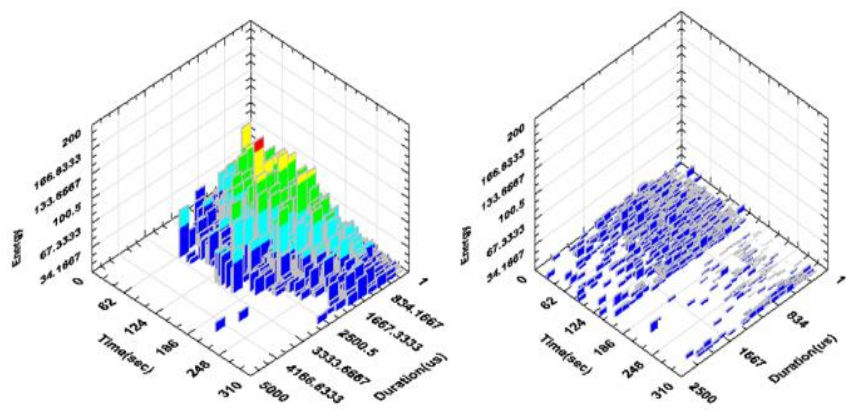

Figure 7. PAC-energy from the sensors while the fault was 90 degrees apart from the top sensor

The results of variable speed tests indicate the sensitivity of AE count to the shaft speed as shown in Figure 8 where the speed was gradually changed from 0 to $300 \mathrm{rpm}$ and dropped back to 0 .

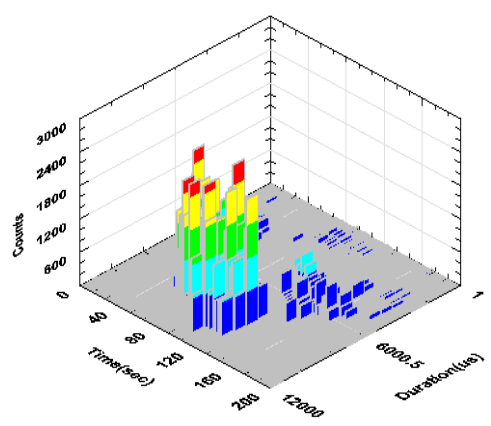

Figure 8. Dependence of AE parameters to the shaft speed

\section{RESULTS AND DISCUSSION}

\subsection{Categorical data analysis}

The data set for the categorical data analysis consisted of data from six bearings: one faulty bearing, two used bearing and three new bearings. The cumulative energy (CE) was 
selected as the response variable. The objective of this categorical analysis is to reveal the effects of explanatory variables (i.e. bearing, speed and unbalance) on the response variable. Due to time limit, it was not possible to perform all the loading patterns for the speed level of 150 and $450 \mathrm{rpm}$. Although, this can be a drawback for the categorical analysis, the results are satisfactory. The GENMOD procedure in SAS was used to conduct the statistical tests. This procedure fits a GLM to the data by maximum likelihood estimation. Through an iterative fitting process, the parameters of the model are numerically estimated in this procedure. Based on the asymptotic normality of maximum likelihood estimators, standard errors, and pvalues of the estimated parameters are computed. The parameter with the smallest p-value will be the most significant parameter in that category. The GENMOD procedure provides a number of probability distributions and link functions such as log-link function.

\subsubsection{Analysis of AE data with various speed levels}

The categorical data analysis (CDA) shows that speed has the most significant effect among the explanatory variables. The bearing type has the least significant effect with the pvalue of 0.6295 (Table 2). Figure 6 supports the SAS results, that speed would positively affect the number of count and the energy of the signal. The interaction between explanatory variables (speed, unbalance and bearing) is added to the model. This 3-way interaction is significant ( $\mathrm{p}$ value $=0.1041)$. This model shows that speed and unbalance have significant effects on the CE level (Table 3).

\begin{tabular}{|c|c|c|c|}
\hline Parameter & Estimate & $\begin{array}{c}\text { Standard } \\
\text { Error }\end{array}$ & Pr > Chi Sq. \\
\hline intercept & 0.1278 & 0.3054 & 0.6757 \\
\hline bearing & -0.0475 & 0.0985 & 0.6295 \\
\hline speed & 0.0011 & 0.0007 & 0.1254 \\
\hline unbalance & 0.0336 & 0.0493 & 0.4962 \\
\hline
\end{tabular}

Table 2. CDA- Model 1

\begin{tabular}{|c|c|c|c|}
\hline Parameter & Estimate & $\begin{array}{c}\text { Standard } \\
\text { Error }\end{array}$ & Pr > Chi Sq. \\
\hline intercept & -0.6358 & 0.5584 & 0.2549 \\
\hline bearing & 0.1680 & 0.1617 & 0.2987 \\
\hline speed & 0.0022 & 0.0010 & 0.0229 \\
\hline unbalance & 0.1717 & 0.0971 & 0.0769 \\
\hline Interaction & -0.0002 & 0.0002 & 0.1041 \\
\hline
\end{tabular}

Table 3. CDA-Model 1 with 3-way interaction

A new model was built based on the interactions between every two explanatory variables (Table 4). The most significant interaction is the bearing-unbalance. In this case, unbalance type is the most significant variable. The effect of categories of each variable on the $\mathrm{AE}$ generation was then investigated. One category was selected as the baseline and the significance of the other categories were analyzed. For speed, $450 \mathrm{rpm}$ was selected as the baseline. As expected, the results indicate that this speed level is more significant than the other two levels. The bearing type 3 (i.e. faulty) was the baseline to analyze bearing types. As shown in the Table 5, type 1 (new bearing) and 2 (used bearing) are not significant compared to the faulty bearing. For the unbalance, type 5 was the baseline. As shown in the Table 6 , type 1 and 2 are not significant at all compared to the couple unbalance i.e. type 5. Interestingly, the SAS output results indicate that couple unbalance is the most significant type of unbalance in comparison with type 3 and 4 . This result is very similar to the graphical balancing calculation that shows couple unbalance needs the highest amount of balancing mass.

\begin{tabular}{|c|c|c|c|}
\hline Parameter & Estimate & $\begin{array}{c}\text { Standard } \\
\text { Error }\end{array}$ & Pr > Chi Sq. \\
\hline intercept & -1.0091 & 0.9006 & 0.2625 \\
\hline bearing & 0.0924 & 0.3834 & 0.8095 \\
\hline speed & 0.0013 & 0.0025 & 0.6070 \\
\hline unbalance & 0.6373 & 0.2128 & 0.0027 \\
\hline bearing $*$ speed & 0.0013 & 0.0010 & 0.1604 \\
\hline bearing*unbal. & -0.1836 & 0.0679 & 0.0069 \\
\hline speed $_{*}$ unbal. & -0.0008 & 0.0005 & 0.1094 \\
\hline
\end{tabular}

Table 4. CDA - Model 1 with 2-way interactions

\begin{tabular}{|c|c|c|c|c|}
\hline Parameter & \multirow{E}{*}{} & Estimate & $\begin{array}{c}\text { Standard } \\
\text { Error }\end{array}$ & Pr > Chi Sq. \\
\hline intercept & & 0.1088 & 0.3034 & 0.7199 \\
\hline bearing & 1 & 0.0101 & 0.199 & 0.9593 \\
\hline bearing & 2 & -0.2117 & 0.2195 & 0.3347 \\
\hline speed & & 0.0011 & 0.0007 & 0.1256 \\
\hline unbalance & & 0.0338 & 0.0493 & 0.4928 \\
\hline
\end{tabular}

Table 5. CDA - Bearing 3 is the baseline

\begin{tabular}{|c|c|c|c|c|}
\hline Parameter & \multirow{2}{*}{ Estimate } & $\begin{array}{c}\text { Standard } \\
\text { Error }\end{array}$ & Pr > Chi Sq. \\
\hline intercept & & 0.2736 & 0.4105 & 0.505 \\
\hline bearing & & -0.0421 & 0.0986 & 0.6696 \\
\hline speed & & 0.0009 & 0.0009 & 0.3432 \\
\hline unbalance & 1 & 0.0808 & 0.214 & 0.7058 \\
\hline unbalance & 2 & -0.2115 & 0.2814 & 0.4521 \\
\hline unbalance & 3 & -0.4037 & 0.2917 & 0.1664 \\
\hline unbalance & 4 & 0.3487 & 0.251 & 0.1648 \\
\hline
\end{tabular}

Table 6: CDA - Unbalance type 5 is the baseline

\subsubsection{Analysis of AE data without the effect of speed}

Since it was realized that speed has the most significant effect on CE, the next set of statistical tests were conducted with the speed level of $300 \mathrm{rpm}$. Having more data was the reason for selecting this speed level. The results of this data analysis indicated that unbalance is more significant in the 
absence of speed effect (Table 7). Similar to the case with various speeds, adding the interactions of these variables lead to the results that all the inputs are significant.

\begin{tabular}{|c|c|c|c|}
\hline Parameter & Estimate & $\begin{array}{c}\text { Standard } \\
\text { Error }\end{array}$ & Pr $>$ Chi Sq \\
\hline intercept & 0.4184 & 0.3326 & 0.2084 \\
\hline bearing & -0.1906 & 0.1393 & 0.1712 \\
\hline unbalance & 0.1362 & 0.0706 & 0.0538 \\
\hline
\end{tabular}

Table 7: Categorical data analysis without the effect of speed

\subsection{ZIP model}

The independent variables in this study were shaft speed and unbalance. The amount of mass required for balancing was used as the unbalance values in the ZIP model. The graphical balancing techniques were utilized to calculate the amount of the balancing mass. The procedure of balancing considers both the balance of forces and couples. It is to be noted that couple unbalance (type 5) needs the highest amount of mass for balancing.

The COUNTREG procedure in SAS was used to develop the ZIP models. This procedure performs nonlinear optimization. Two iterative minimization method were applied; (1) the quasi-Newton method and (2) the NewtonRaphson method. The ZIP models provide the probability (p) of obtaining zeros and the parameter $(\lambda)$ for the Poisson model. The probability of obtaining zero for the faulty bearing is almost zero for all the tests. This implies the existence of strong burst signal in all the AE hits. To provide a fair comparison, Figure 9 shows the $\lambda$ for different unbalance types at the speed level of $300 \mathrm{rpm}$.

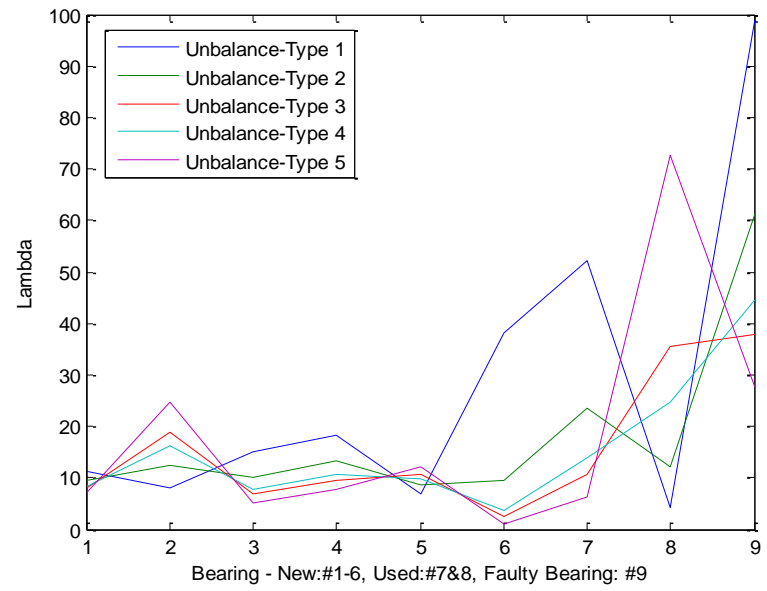

Figure 9. The Lambda from the ZIP model for different unbalance type at $300 \mathrm{rpm}$

In this graph, bearing 9 represents the faulty bearing; bearing 7 and 8 represent the used bearings. It is clear that the highest values of $\lambda$ belong to either used or the faulty bearings. It implies that these bearing generate stronger burst signals in comparison with all the six new bearings used in this study. It is interesting to note that the highest $\lambda$ $(=429)$ was obtained for the couple unbalance of the faulty bearing.

In order to provide a platform for diagnostics, cumulative sum (CUSUM) chart was utilized (He et al., 2011, Montgomery2001, Leger et al., 1998). This chart detects the deviation of the process mean through cumulative sums of the shift between sample averages from a target value. CUSUM charts are sensitive to small and moderate changes in the process mean. Such changes, e.g. one-sigma shift in the mean, are hardly detectable by Shewhart-charts. The run chart of CUSUM displays the successive differences between the sample average and the target i.e. process mean. The two-sided tabular CUSUM chart is defined by

$S^{ \pm}(n)\left\{\begin{array}{cc}0, & \text { if } n=0 \\ \max \left\{0, S^{ \pm}(n-1) \pm\left(x_{n}-\hat{\mu}_{0}\right)-k \hat{\sigma}_{x}\right\} & \text { if } n=1,2, \ldots\end{array}\right.$

where $x_{n}=\lambda_{n}$ (or $\left.p_{n}\right), S^{+}(n)$ and $S^{-}(n)$ give the cumulation on high side and low side respectively and $\mathrm{k}$ is the threshold for cumulation which is also called allowable slack. This parameter is the minimum difference between the target and sample average. We have $\mathrm{H}=h \sigma_{x}=\tan (\theta) d \sigma_{x}$ as the decision interval and $\mathrm{h}$ is called the decision parameter as shown below.

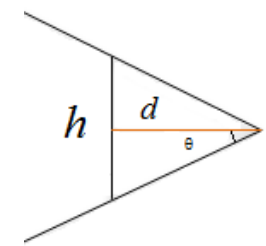

If $S^{+}(n)$ or $S^{-}(n)$ exceeds $H$, the process is out- of-control. Figures 10 and 11 show the two-sided CUSUM charts of $\lambda$ and $p$ for the case when only the new bearings were used. Here, as none of the plotted points cross the arms of the Vmask we conclude that the process is in control.

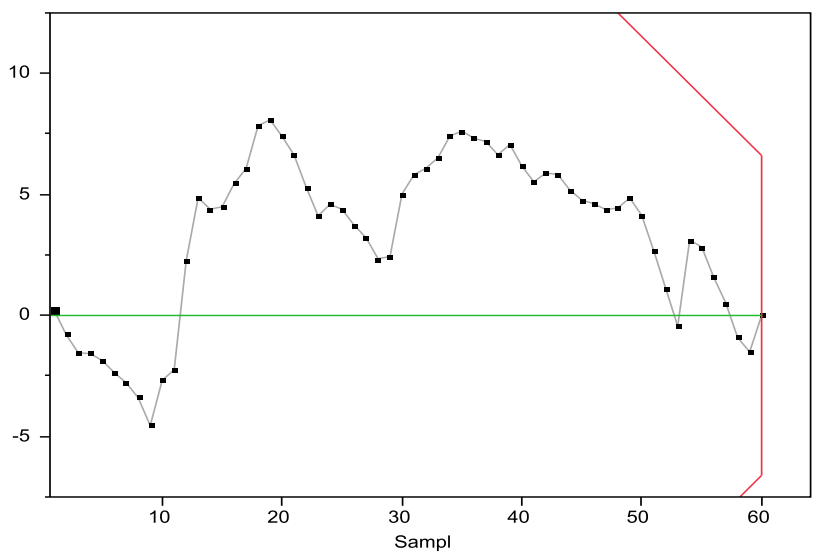

Figure 10. CUSUM charts of $\lambda$ for new bearings 


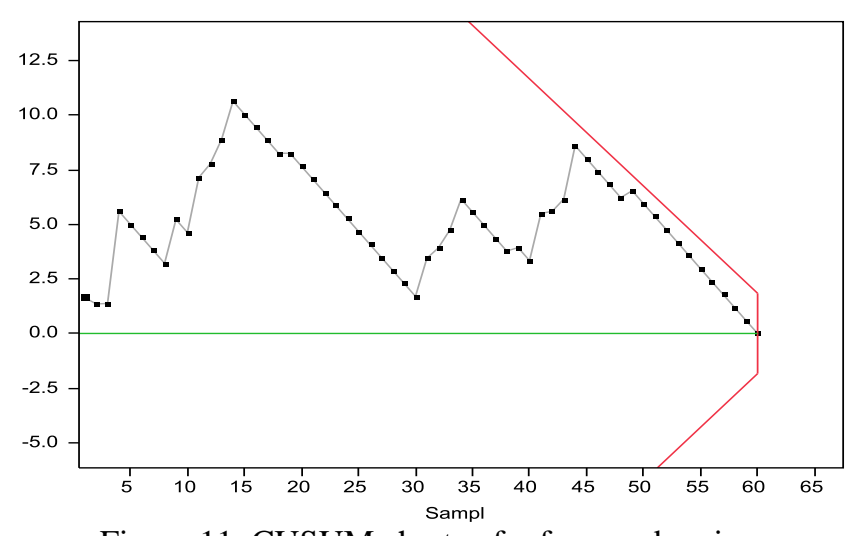

Figure 11. CUSUM charts of $p$ for new bearings

Figures 12 and 13 show the CUSUM charts of $\lambda$ and $p$ for the case when used bearings were also taken into account. The chart for $\lambda$ clearly shows that the bearings experienced certain deviation and they are not as good as new bearings.

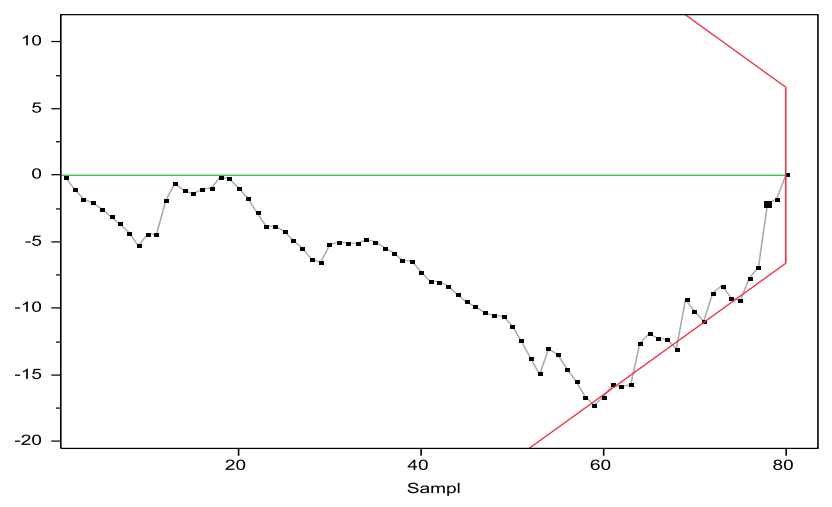

Figure 12. CUSUM charts of $\lambda$ for new and used bearings

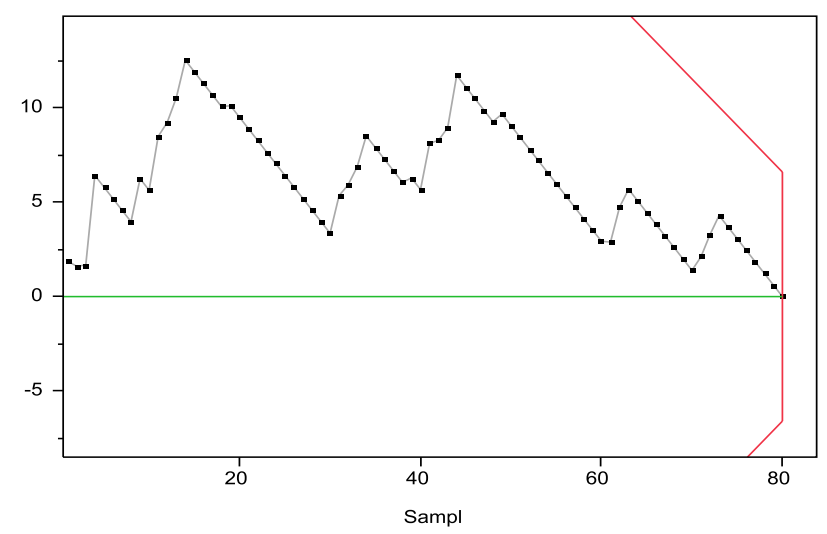

Figure 13. CUSUM charts of $p$ for new and used bearings

Figures 14 and 15 show the CUSUM charts of $\lambda$ and $p$ for the case when all types of bearings were taken into account. These charts clearly depict the out of control condition.

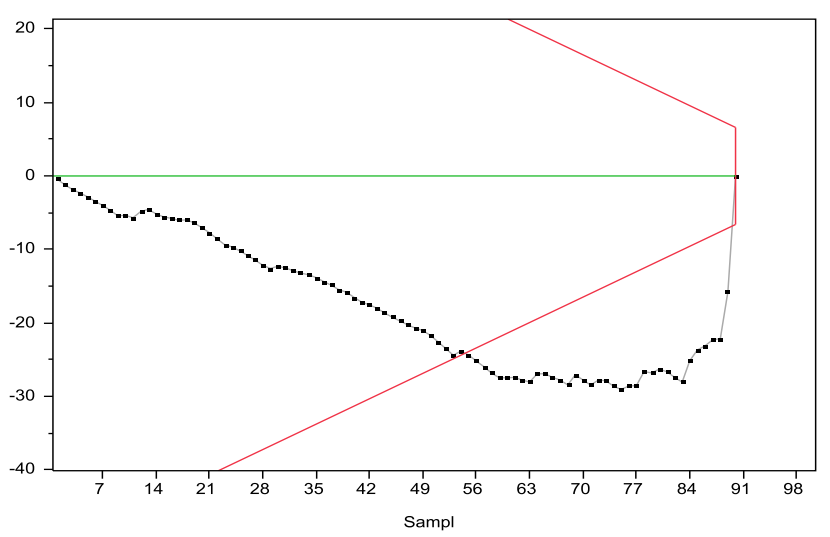

Figure 14. CUSUM charts of $\lambda$ for all bearings

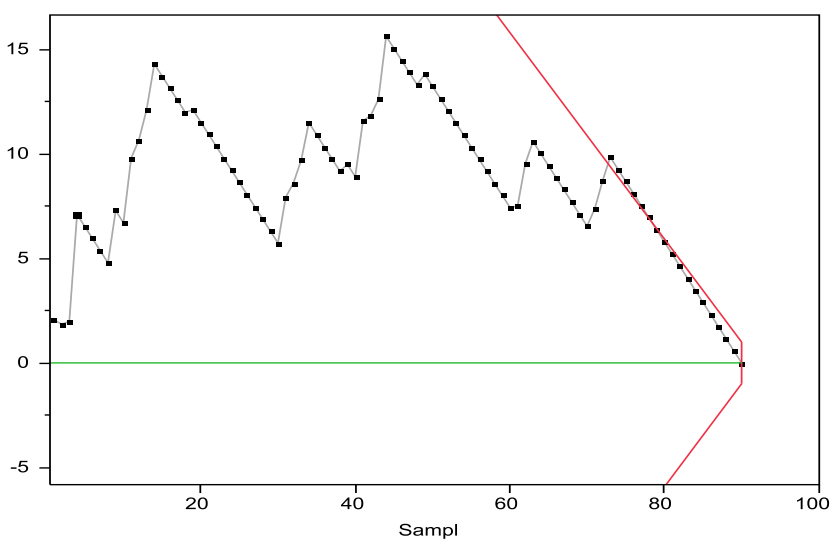

Figure 15. CUSUM charts of $p$ for all bearings

\section{Conclusion}

This paper investigates the usefulness of AE data for unbalance analysis in rotary systems. The parameter of interest in this study is the PAC-energy which covers the significance of count, duration and peak amplitude in $\mathrm{AE}$ signals. The categorical data analysis based on generalized linear models is presented. The results of this analysis confirm the visual observations of the AE signals during the data acquisition process. It was discovered that speed has the most significant effect on PAC-energy. The results of the categorical analysis indicated that loading patterns with two disks were more significant. More importantly, the results specified the significance of couple unbalance. In addition, it was also understood that bearing types influence the level of cumulative energy. It was realized that the faulty bearing is the most significant in producing more PACenergy. Interestingly, the effect of used bearing was more significant than new bearings in this respect.

A statistical model based on Zero-Inflated Poisson regression is presented to handle over-dispersion and excess zeros of the counting data. Combined with CUSUM chart, the ZIP model can provide a platform to diagnose the faults in bearings. It was observed that CUSUM charts of both $\lambda$ and $p$ can be utilized for fault detection and also to track the 
health of bearings. It was also observed that while unbalance influences the amplitude of signal, speed directly affects the probability of getting more zero counts.

For more effective diagnosis, the pattern of control charts can be recognized through artificial intelligence methods. Moreover, distribution-free tabular CUSUM chart can be investigated. It is noteworthy to point that $\mathrm{AE}$ can be utilized to detect and quantify unbalance in the rotary systems. However, it is essential to consider the sources of uncertainty such as the instrumentation errors, rotor system nonlinearities, stiffness (rotor, bearing support and foundation), and the nature of bearings.

\section{REFERENCES}

Tan, C., Irving, P., Mba, D. (2007). A comparative experimental study on the diagnostic and prognostic capabilities of acoustics emission, vibration and spectrometric oil analysis for spur gears. Mechanical Systems and Signal Processing, vol. 21, pp. 208-233.

Alghamd, A., \& Mba, D. (2006). A comparative experimental study on the use of acoustic emission and vibration analysis for bearing defect identification and estimation of defect size. Mechanical Systems and Signal Processin, vol. 20, pp. 1537-1571.

Tandon, N., \& Mata, S. (1999). Detection of Defects in Gears by Acoustic Emission Measurements. Journal of Acoustic Emission, vol. 17, pp. 23-27.

Eftekharnejad, B., \& Mba, M. (2009). Seeded fault detection on helical gears with acoustic emission. Applied Acoustics, vol. 70, pp. 547-555.

Mba, D., \& Rao, R. B. K. N. (2006). Development of Acoustic Emission Technology for Condition Monitoring and Diagnosis of Rotating Machines; Bearings, Pumps, Gearboxes, Engines and Rotating Structures. The Shock and Vibration Digest, vol. 38, pp. 3-16.

Yoshioka, T., \& Fujiwara, T., (1982). New acoustic emission source locating system for the study of rolling contact fatigue. Wear, vol. 81, pp. 183-186.

Niknam, S. A. \& Liao, H. (2011). Diagnostic and Prognostic Modeling of High-Speed Milling Machine Cutters. The 7 th International Conference on Mathematical Methods in Reliability - Theory, Methods, Applications, Beijing, China.

PAC (Physical Acoustic Corporation) (2007). PCI-2 Based AE System. Princeton Jct, NJ

Miyachika, K., Oda, S., Koide, T. (1995). Acoustic Emission of Bending Fatigue Process of Spur Gear Teeth. Journal of Acoustic Emission, vol. 13, pp. 47-53.

He, D., Li, R., Bechhoefer, E. (2010). Split Torque Type Gearbox Fault Detection using Acoustic Emission and Vibration Sensors. International Conference on Networking Sensing and Control.

Bansal, V., Gupta, B. C., Prakash, A., Eshwar, V. A. (1990). Quality inspection of rolling element bearing using acousic emission technique. Acoustic Emission, vol. 9, pp. 142-146.

Choudhury, A. \& Tandon, N. (2000). Application of acoustic emission technique for the detection of defects in rolling element bearings. Tribology International, vol. 33, pp. 39-45.

Lu, B., Y. Li, Wu, X., Yang, Z. ( 2009). A review of recent advances in wind turbine condition monitoring and fault diagnosis. Power Electronics and Machines in Wind Applications, Milwaukee, WI.

Hyers, R. W., McGowan, J. G., Sullivan, K.L., Manwell, J.F., Syrett, B.C. (2006). Condition monitoring and prognosis of utility scale wind turbines. Energy Materials, vol. 1, pp. 187-203.

Hameed, Z., Hong, Y.S., Cho, Y.M., Ahn, S.H., Song, C.K. (2009). "Condition monitoring and fault detection of wind turbines and related algorithms: A review. Renewable and Sustainable Energy Reviews, vol. 13, pp. 1-39.

Tandon, N., \& Nakra, B. C. (1990). Defect Detection of Rolling Element Bearings by Acoustic Emission Method. Journal of Acoustic Emission, vol. 9, pp. 2528.

Morhain, A., \& Mba, D. (2003). Bearing defect diagnosis and acoustic emission. Engineering Tribology, vol. 217, pp. 257-272.

X. Li (2002). A brief review- acoustic emission method for tool wear monitoring during turning. International Journal of Machine Tools \& Manufacture, vol. 42, pp. 157-165.

Sharma, V.S., Sharma, S. K., Sharma, A. K. (2007). An approach for condition monitoring of a turning tool. Journal of Engineering Manufacture, vol. 22, pp. 635646.

Carpinteri, A., Lacidogna, G., Pugno, N. (2007). Structural damage diagnosis and life-time assessment by acoustic emission monitoring. Engineering Fracture Mechanics, vol. 74, pp. 273-289.

Liu, W.S., \& Cela, J. (2008). Count Data Models in SAS. SAS Global Forum.

Lambert, D. (1992). Zero-Inflated Poissoin Regression, with an application to defects in manufacturing. Technometrics, vol. 34, pp. 1-14.

Guikema, S. D. \& J. P. Coffelt (2008). Modeling count data in risk analysis and reliability engineering. In K. B. Misra (ed.). Handbook of Performability Engineering, London, Springer.

Wowk V. (1995). Machinery Vibration: Balancing. New York, McGraw-Hill.

He, S., Huang, W., Woodall, W.H . (2011). CUSUM Charts for Monitoring a Zero-inflated Poisson Process. Quality and Reliability Engineering International, vol. 28, pp. 181-192.

Montgomery, D. C. (2001). Introduction to Statistical Quality Control, 4th ed. John Wiley \& Sons, New York. 
Leger, R.P., W.J. Garland, and W.F.S. Poehlman, (1998). Fault detection and diagnosis using statistical control charts and artificial neural networks. Artificial Intelligence in Engineering, vol. 12, pp. 35-47.

\section{BIOGRAPHIES}

Seyed A. Niknam received the B.Sc. degree in mechanical engineering in 2003, the M.Sc. degree in advanced manufacturing systems from Brunel University in 2006, and the M.Sc. degree in reliability and maintainability engineering from the University of Tennessee, Knoxville in 2011. Currently, he is a PhD candidate in the department of Industrial and Systems Engineering at the University of Tennessee, Knoxville. He has been working as the instructor of the core courses in the reliability and maintainability engineering program since January 2012. His research interests include reliability of repairable systems, diagnostics and prognostics.

Tomcy Thomas received his B.E. (Electronics) from Marathwada University, India in 1991. He received his MBA (Operations Management) from IGNO University, India in 2000. He received his MS (Engineering Management) from the University of Tennessee in 2010. He is currently a Ph.D. candidate in the Department of Industrial and Systems Engineering at the University of Tennessee, Knoxville. His research interests include energy management, reliability engineering and supply chain optimization.

Dr. J. Wesley Hines is currently the Nuclear Engineering Department Head at the University of Tennessee. He has served as Interim Associate Dean for Research in the College of Engineering and more recently as Interim Vice Chancellor for Research for the Campus. He received the BS degree in Electrical Engineering from Ohio University in 1985, and then served as a nuclear qualified submarine officer in the US Navy. He later received both an MBA and an MS in Nuclear Engineering from The Ohio State University in 1992, and a Ph.D. in Nuclear Engineering from The Ohio State University in 1994. He has been with the University for 18 years. Dr. Hines teaches and conducts research in artificial intelligence and advanced statistical techniques applied to process diagnostics, condition based maintenance, and prognostics; and has made notable accomplishments in the invention and development of reliability enhancing condition monitoring technologies. These technologies have allowed operators to understand the condition of vital assets and in several cases to predict their remaining useful life with quantifiable confidence bounds. He has authored over 275 papers and has three patents that have been implemented in commercial products.
Dr. Rapinder Sawhney has been a faculty member with the Department of Industrial and Systems Engineering (ISE) for almost two decades. He was named as head of ISE and Weston Fulton Professor in 2010. He is also a faculty member of the Center for Interdisciplinary Research and Graduate Education (CIRE), a joint effort between UT and ORNL focused on renewable energy. Sawhney received his Ph.D in engineering science and mechanics from the University of Tennessee. He is a member of the Institute for Industrial Engineers and the American Society of Quality and is also a committee member for the Boeing Welliver Fellowship Committee and the Institute of Industrial Engineers Lean Division. 